\title{
Low-dose radiation cancer risk hypothesis may lead to 'radiophobia'-driven imaging avoidance?
}

Dear Sir,

We read with great interest the recent expert consensus document on the optimal use of ionizing radiation in cardiovascular imaging. ${ }^{1}$ Radiation-induced cancer is a stochastic event and its likelihood is assumed to increase approximately linearly with dose. ${ }^{1}$ However, the assessment of risk, associated with the performance of low-dose diagnostic cardiovascular procedures (significantly lower than $100 \mathrm{mSv}$ ), is based on data that refer to much higher doses, using mathematical extrapolation models. ${ }^{2}$ Interestingly, it should be noted that a proposed model (the hormesis model) implies lower subsequent cancer risk in individuals exposed to low doses. $^{2}$

Despite the inconclusive evidence regarding the potential adverse effects in the low-dose burden of nuclear cardiology procedures, we often face strong concerns over the radiation safety during our clinical routine. Commonly, patients and their relatives are troubled for the radiation-induced health consequences, and in particular the possibility of induced cancer risk. Similarly, referring physicians have to deal with such concerns when ordering a diagnostic exam that employs ionizing radiation. Moreover, personnel working in departments other than nuclear medicine are often troubled due to possible radiation exposure during the patient care after a nuclear cardiology procedure.

Radiation hazards are occasionally presented to the public through mass media, including social media, possibly leading to an overestimated radiation fear deeply ingrained in the public psyche. The truth, however, is that the health risk posed by diagnostic ionizing radiation is nowhere near as great as commonly assumed, mainly based on the invalidated linear no- threshold hypothesis (LNTH). ${ }^{3}$ We believe that cardiovascular specialists should be well aware of the nonexistent credible evidence of cancer risk from diagnostic imaging procedures, in order to accurately assess the risk-benefit relationship and provide the required objective information, not only to the patients and their relatives but also to other healthcare professionals. Otherwise, we believe that there is a potential risk of (a) under-usage of beneficial diagnostic procedures, (b) patient's 'radiophobia'-driven imaging avoidance, and (c) physician-recommended substitution of alternative procedures due to non-evidence-based fear of low-dose radiation.

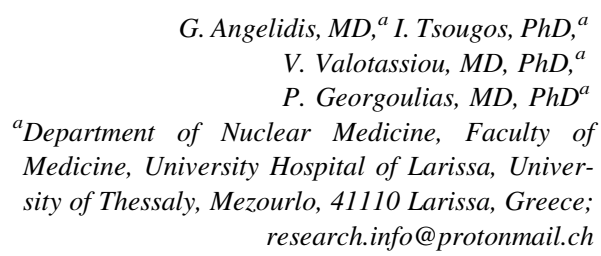

\section{References}

1. Hirshfeld JW Jr, Ferrari VA, Bengel FM, Bergersen L, Chambers CE, Einstein AJ, et al. 2018 ACC/HRS/NASCI/SCAI/SCCT expert consensus document on optimal use of ionizing radiation in cardiovascular imaging: best practices for safety and effectiveness: A report of the american college of cardiology task force on expert consensus decision pathways. J Am Coll Cardiol 2018;71:283-351.

2. Zanzonico P, Dauer L, Strauss HW. Radiobiology in cardiovascular imaging. JACC Cardiovasc Imaging 2016;9:1446-61.

3. Siegel JA, Pennington CW, Sacks B. Subjecting radiologic imaging to the linear no-threshold hypothesis: A non sequitur of non-trivial proportion. J Nucl Med 2017;58:1-6.

doi:10.1007/s12350-018-1354-0 